\title{
Arquitectónica de la Ciudad Aumentada. Distensión, extensión, sintonización
}

\author{
Eduardo RoIG \\ Departamento de Arquitectura \\ Universidad Antonio de Nebrija \\ comboroig@gmail.com
}

Recibido: $29 / 08 / 2014$

Modificado: $28 / 11 / 2014$

Aceptado: $01 / 12 / 2014$

\section{Resumen}

La emergencia del entorno aumentado, producto del solapamiento de la capa digital en el entorno físico, induce cambios profundos en la ciudad y en sus ciudadanos. La inserción de esta dimensión digital impone la modificación de los límites dicotómicos que tradicionalmente han segregado la estructura urbana. El usuario sintoniza la Ciudad Aumentada acomodando la estructura topológica de la nueva extensión electrónica a sus hábitos de vida. Este proceso de simbiosis de realidades produce nuevos paisajes urbanos cuya cartografía arquitectónica precisa de neologismos que acuñen y categoricen aquellos conceptos generados por la Ciudad Aumentada.

Palabras clave: entorno, aumentado, digital, dicotomía, ciudad.

Title: Architectonics of the Augmented City. Distension, extension, tuning

\section{Abstract}

The emergence of the augmented environment, where the digital layer overlaps the physical environment, procures deep changes in the city and in its inhabitants. The insertion of this digital dimension varies the dichotomous boundaries in which the urban structure has been traditionally segregated. The user tunes the Augmented City fitting the topological structure of the new electronic extension. This process of reality symbiosis produces new landscapes, whose architectonic cartography demands a new language to name and to categorize those concepts generated by the Augmented City.

Keywords: environment, augmented, digital, dychotomies, city.

\section{Índice}

1. Distensión del límite dicotómico en la Ciudad Aumentada

2. Extensión de la ciudad edificada: la hiperciudad

3. Sintonización del paisaje urbano. La ciudad dial y el umbral aumentado 


\section{Distensión del límite dicotómico en la Ciudad Aumentada}
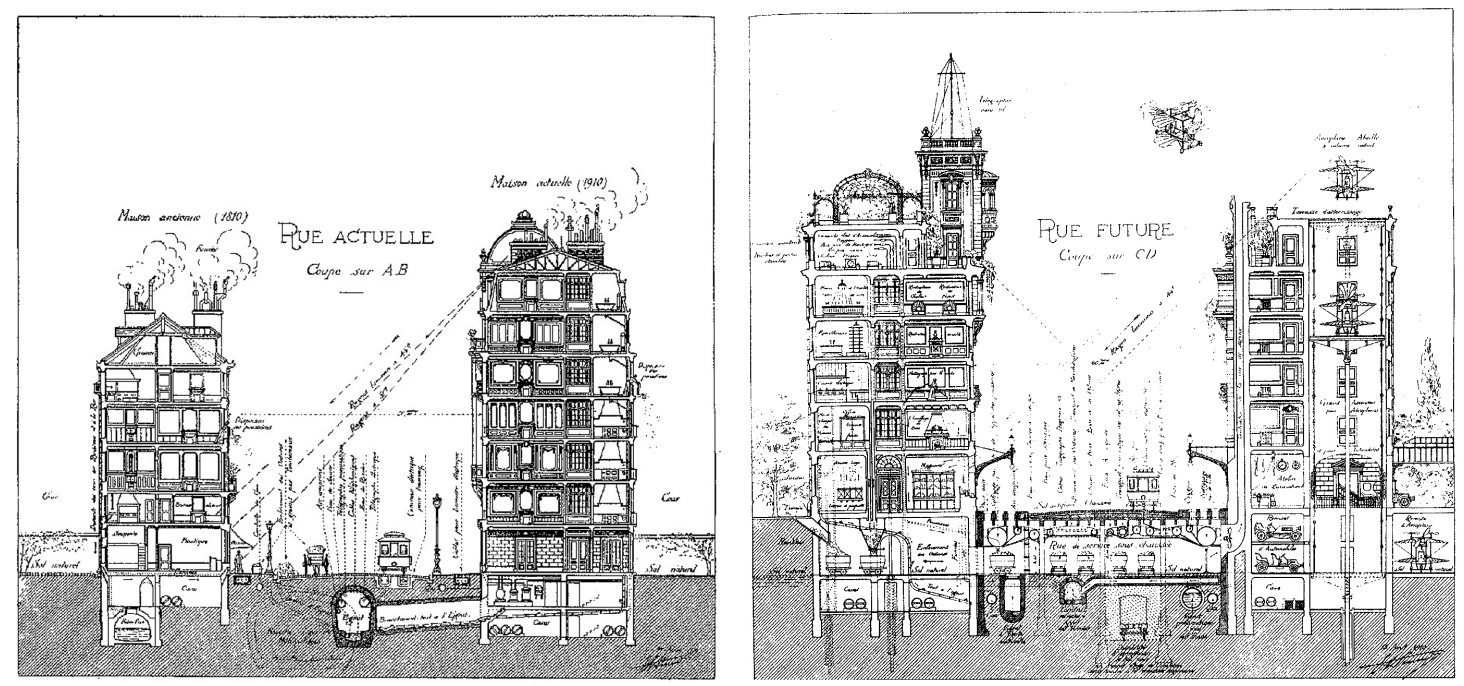

Figura 1. Eugène Hénard, Rue Actuelle, Rue Future, 1910.

¿Cómo debe ser la ciudad del futuro?

Asociada tradicionalmente al espacio público confinado entre inmuebles privados, la calle sigue siendo para muchos la viva imagen de la ciudad, su referente histórico más artificial, su alter ego. Preguntado en 1910 por la calle del futuro, Eugène Hénard respondió con la Rue Future (figura 1), una representación de un pronóstico cuya sección estructuraba un solapamiento de los usos, de los sistemas energéticos y de las redes de transporte. El plano dejaba intuir casi telegráficamente la segregación dicotómica entre la cosa pública y el dominio privado ${ }^{1}$. La Rue Future exhibía las aportaciones técnicas que iban a resolver los problemas de insalubridad propios de los desarrollos urbanos de la Revolución Industrial. Los avances tecnológicos de aquella primera época de la máquina configuraron en el pensamiento de Hénard el perfil de una calle que planteaba la estratificación vertical del sistema de circulación y la mencionada separación de usos públicos y privados. La circulación de vehículos rodados resultaba un problema de difícil solución en aquellos momentos, aunque también lo fuera en la Roma imperial o en el burgo ciudad medieval. Hénard anticipó el estacionamiento en la cubierta de vehículos voladores ${ }^{2}$ e integró en el subsuelo las instalaciones servidoras y el tránsito pesado de mercancías y otros flujos -a excepción del tranvía superficial-. Hénard orquestó un proyecto de una ciudad del futuro fundamentada en la capacidad de

${ }^{1}$ Alguna vez oí al profesor Antonio Miranda (ETSAM) referirse en estos términos a la dimensión del ancho de la calle, tal que un peatón pudiera distinguir nítidamente el contenido de los escaparates de la acera de enfrente.

${ }^{2}$ Esa misma hipótesis desencadena el proyecto Skycar City (MVRDV-UWM 2007). 
las nuevas técnicas para proponer un nuevo escenario más eficiente y salubre: una apuesta evidente por el progreso.

Un siglo después, la segregación de los usos característica de la ciudad de Eugène Hénard resulta insostenible ${ }^{3}$. La calle como elemento referencial del tejido urbano comienza en este siglo XXI a ser puesta en entredicho por los nuevos modelos asociativos entre lo público y lo privado. La definición del límite que tradicionalmente separaba las dicotomías privado-público, local-global, individualcolectivo y tantas otras propias en la trama urbana -incluyendo probablemente la más determinante casa-ciudad- queda obsoleta en la Ciudad Aumentada, que se construye o construimos en el solapamiento de la capa digital con la ciudad tradicional. La relación de vasos comunicantes que históricamente ha regulado lo privado y lo público resulta con frecuencia poco operativa e irreal en la ciudad contemporánea. Al ser el entorno aumentado de la ciudad homónima la suma del entorno físico y el entorno digital ${ }^{4}$, es factible que uno de ellos corresponda a un ámbito privado y otro a uno público, lo cual supone un contrasentido de difícil legislación. Así, el corpus del planeamiento urbano ortodoxo vigente en el siglo XX ha estimado casi sin excepción que un lugar con un uso asignado no puede ser privado y público a la vez ${ }^{5}$.

Aristóteles demostró la existencia indisoluble de los conceptos dicotómicos. Éstos siguen vigentes en la Ciudad Aumentada, pero asumen cambios categóricos y semánticos que desfiguran la divisoria del par dicotómico. Surgen así resquicios neutros entre los conceptos opuestos, espacios que requieren un tratamiento cuya capacidad de cambio constituye una atención principal del proyecto. El modelo de ciudad holística, compacta, compleja, eficiente y estable socialmente propuesta por la Agencia de Ecología Urbana de Barcelona (Rueda 2012: 8) como paradigma actual de ciudad sostenible recoge en sus fundamentos esta directriz, al tiempo que sugiere la amortización de las sinergias derivadas de la hibridación programática de usos como elemento enriquecedor de la trama urbana. Los no-lugares, los lugares in between o inter-medios y otras muchas demarcaciones cuyo uso, geometría o cualidad han sido desdibujados a favor de otras cualidades son un claro ejemplo de espacios neutros. En palabras de Marc Augé6 (1995: 77):

\footnotetext{
${ }^{3}$ Los efectos del Plan General de Madrid de 1997 son demoledores en este sentido.

4 En el entorno aumentado, el dominio digital se encuentra referenciado en el entorno físico o biológico a través de mediadores localizadores o locative media. Esta tecnología (GPS, códigos QR, etc.) georreferencia la información digital en lugares concretos. Las Google Glass son un buen ejemplo de ello.

5 Un individuo puede encontrarse en un parque público o en el metro inmerso en una videoconferencia privada.

${ }^{6}$ Acuñó el término "no-lugar".
} 
Si un lugar puede definirse como un lugar de identidad, relacional e histórico, un espacio que no puede definirse como espacio de identidad, ni como relacional, ni como histórico, definirá un no-lugar.

La coyuntura alcanza a todas las dicotomías cuyos conceptos o acciones complementarias participen del entorno digital de la ciudad. La contaminación de sus límites es producto de una acción simétrica y simultánea: por un lado, la "ciudadanización" de la vivienda (domus); por otro, la "domesticación" de la ciudad. El cambio acelerado que las TIC han inferido a la mutación del criterio relacional entre lo público y lo privado se encuentra entre las causas más determinantes de este proceso de transformación de la ciudad.

Este argumento resulta alineado con lo que algunos han señalado como "el declive del espacio público", apuntado prematuramente por el sociólogo Richard Sennett (1970), cuya tesis avanza el desplazamiento de los asuntos públicos a la esfera privada y la "ocupación" de lo público por asuntos privados. La dislocación del límite entre programas públicos y privados también es analizada por Zygmunt Bauman (2001):

[...] un aspecto paralelo al tipo de globalización económica neoliberal dominante, y hace notar la desaparición de los espacios públicos tradicionales, espacios de discusión donde se genera el sentido y se negocian los significados, sustituidos por espacios de creación privada destinados a ser objeto de consumo, consecuencia de la paridad social generada por la globalización, que a unos hace locales y a otros globales. (Vidal y Pol 2005: 281)

La segregación social genera lo que Trevor Boddy (2004) denominó ciudad análoga ${ }^{7}$, entendida como un simulacro o analogía de la ciudad porque descuida la civitas y la polis ${ }^{8}$. Este modelo de ciudad construido sobre un loci aumentado, es decir, con una nueva extensión dimensional integrada que defina un tejido urbano híbrido, estaría compuesto por un conglomerado de:

${ }^{7}$ Echeverría (1999: 112) se refiere a la analogía en los siguientes términos: "[...] en el E1 (medio ambiente natural) y en el E2 (entorno urbano) se razona por metáfora y analogía. Hasta la geometría está basada en la proporcionalidad, que no es sino una modalidad de analogía [...]. En el tercer entorno las analogías no desaparecen, pero su importancia es mucho menor. El aparato sensorial humano está determinado hasta tal punto por las relaciones de analogía que incluso el mundo digital acaba adoptando un formato analógico para que los seres humanos lo puedan percibir $[\ldots]^{\prime \prime}$.

${ }^{8}$ Para ampliar esta reflexión, véase El espacio público: ciudad y ciudadanía (Borja y Muxí 2001). 
datos con etiquetas geo-referenciadas, notas, blogs, fotografías, videos, sonidos, transacciones [...] que constituyen el material de construcción, adquiriendo orden y significado según se habita. No puede ser vista con el ojo desnudo, por lo que su verdadero carácter está inexorablemente ligado a la tecnología y a la interfaz que facilite su percepción, el agente mediador entre el sujeto y la ciudad. (Matsuda 2010: 12)

Para Matsuda (2010: 12) la ocupación de la Ciudad Aumentada "no será una experiencia desconocida y temerosa, sino personal y doméstica". Cabría preguntarse por las numerosas acepciones del término "personal" que admite el vaticinio. La Ciudad Aumentada es un acontecimiento mediado por los dispositivos electrónicos de cada usuario, cada vez más personales, como lo son también los contenidos que la edifican. La domesticidad es ciertamente una propiedad inherente al ser humano que trata de colonizar su proximidad, proyecta su identidad en el coche, en su casa, en su trabajo, en sus hijos, etc., pero también en los medios. A pesar de estar desaconsejado en muchas ocasiones, éste continúa nutriendo las redes sociales con su privacidad y habitualmente también con su intimidad. Compartir cada vez más y mejor parece ser la tendencia.

El agotamiento general de la extensión dicotómica que categoriza los espacios históricos y la aparición de entidades espaciales que responden a nuevas cualidades híbridas demandan urgentemente términos que definan los nuevos conceptos y acuñen las acciones y los sustantivos que construyen el entorno aumentado. Gran parte de su vocabulario se originó en los tiempos heroicos de la invención de la computación. Pero al concluir la Revolución Digital (Negroponte 1998) y ganar velocidad el proceso de construcción del entorno aumentado, el campo teórico de la crítica y el mercado de la producción tecnológica ${ }^{9}$ comenzaron a alimentar con mayor celeridad si cabe la sustantivación del nuevo entorno. En Los Señores del aire: Telépolis y el Tercer Entorno, Echeverría (1999: 80) teoriza sobre este particular que nos concierne y propone -en oposición a la terminología pre-digital anterior a Telépolis- un conjunto de voces que definen propiedades características de los entornos aumentados, del cual cabe extraer las siguientes: "distal", frente a proximal; "multicrónico", frente a sincrónico; "representacional", frente a presencial; o "transnacional", frente a nacional. Del mismo modo,

${ }^{9}$ Su origen anglosajón o asiático hace que los neologismos tecnológicos coincidan casi siempre con anglicismos. Esta cercanía a la invención tecnológica parece advertir en la lengua inglesa una mayor tradición, flexibilidad y capacidad para la creación semántica. Habitualmente, la transferencia de la producción tecnológica al mercado tiene lugar primero en Estados Unidos y, a continuación, el producto se vende en los demás continentes. 
"tecnomadismo"10 o "electronomadismo" (Mitchell 2004: 158) se suman a otras propuestas (Matsuda 2010: 39) que valoran la ocupación "blanda" de los nuevos tecno-lugares. Matsuda sugiere para este glosario de la Ciudad Aumentada una nueva jerarquía dicotómica con ejemplos como "abierto frente a propietario"11, en referencia a la accesibilidad y autoría del entorno aumentado, o "duro" y "blando"12, para distinguir lo objetivo y fijo frente a lo subjetivo y dinámico. Sin duda el lenguaje, que diría Wittgenstein, es un buen método para llegar al conocimiento de la realidad. Sobre todo, cuando ésta apenas está recién inaugurada.

\section{Extensión de la ciudad edificada: la hiperciudad}

Pablo R. acuñó su recuerdo de Venecia como la ciudad soporte de la Bienal de Arte. Era pintor. Cada rincón de la ciudad escondía una acción artística, como si de una red de franquicias se tratara. La Bienal constituía un conjunto heterogéneo de microimpactos, un guante urbano en la mano veneciana que mantenía cautivo al turista. El recuerdo de Pablo M. evocaba Venecia como una confluencia de sabores y olores que cristalizaba en pequeños puestos de pizza, cicatti y polpete. Las calles constituían auténticos circuitos gastronómicos. El mapa sensorial veneciano de Pablo M. comprendía una amalgama de nutrientes recomendado por las guías Lonely Planet, Gourmet Tour y un extenso e-mail remitido por su amiga veneciana Sara Martini. Tal era su interpretación de la ciudad, probablemente consecuencia de una infancia transcurrida entre las mesas del restaurante que aun comandaban sus padres.

Pablo O. se despertaba con el sonido acuático de los taxis y sincronizaba su biorritmo con el tañir del campanile de San Marcos. En sus paseos, se elevaba sobre la reverberación pétrea de las composiciones polifónicas que Antonio Vivaldi orquestó para el conjunto de cámara de la iglesia de la que fue párroco, sobre las interpretaciones corales en San Marcos, el murmullo de la muchedumbre de Rialto, las sirenas de los vaporettos, los ecos de los gondolieri. El mapa de los sonidos de la ciudad ordenaba el recuerdo de la Venecia de Pablo O. (Eduardo Roig)

El cerebro humano, sugestionado por la disposición a priori de la subjetividad, dispone caprichosamente su atención hacia una interpretación particular del conjunto perceptivo. El visitante que deambula por Venecia está predispuesto a proyectar en ella su circunstancia personal. No es verdaderamente la apariencia aséptica

10 Del inglés technomadism. Steve K. Roberts fue el tecnómada pionero, su Nomadic Research Labs está disponible en: http://microship.com/ [Consulta: 10/05/2012].

${ }^{11}$ Del inglés, open vs. proprietary.

${ }^{12}$ Del inglés, soft vs. hard. 
de una confluencia arquitectónica lo que fabrica su experiencia. Parece que pocos son los recuerdos de ciudades cimentados sobre el diagrama infraestructural que las ordena. A este respecto fenomenológico, las palabras de Marshall McLuhan (1967) sentencian: "la ciudad ya no existe, salvo como espejismo cultural para turistas".

La ciudad se erige como una estructura poliédrica de información localizada en el mercado, en los cafés, en la Bolsa, en las plazas, en la comunidad de vecinos, en la vivienda. Las fuentes de información son múltiples: el ciudadano, los sistemas energéticos, la geometría de su traza, la política, etc. Su experiencia se obtiene a través de medios diversos: dispositivos electrónicos, internet, tuberías, aire, carreteras, etc. Esta matriz informacional propicia interacciones en forma de vínculos o disociaciones que se manifiestan en una configuración concreta espacio-temporal de contenidos y continentes, de llenos y/o vacíos. La estructura urbana se acomoda según la correspondencia entre las diferentes categorías de información -aunque a veces se invierte el orden causal de la afirmación- dando forma a una red compuesta por nodos emisores de información -cada ciudadano, cada máquina- y conexiones, las cuales irrigan un tejido con capacidad de atraer o generar actividad nueva.

La ciudad no se agota en su traza construida ${ }^{13}$. Su organización espacial extensa resulta sustituida a diario por otra de índole topológica, donde la distancia en metros no es lo relevante y sí lo es la capacidad informacional del tejido urbano. A esta circunstancia la denomina Manuel Castells "espacio de flujos" (Castells apud Echeverría 1999: 89).

La percepción de la ciudad como red de información se califica habitualmente con la partícula hiper-, en referencia al exceso que opera en el sustantivo prefijado. La hiperciudad excede la realidad presencial del modelo urbano tradicional. De igual modo, el hipertexto desborda al texto. En este sentido, puede afirmarse que la hiperciudad siempre ha existido porque siempre ha denotado información, aunque la coyuntura tecnológica actual alimente el concepto exclusivo de hiperciudad vinculado a la ciudad mediada tecnológicamente. En la Ciudad Aumentada, el medio digital amplía la capacidad informacional del entorno urbano. La acumulación exponencial de información digital responde al proceso dinámico de generación, captura, análisis, visualización y almacenamiento, generada en un $70 \%$ (International Data Corporation 2008) por

13 Sobre este particular reflexiona Echeverría (1999: 89) en el apartado "Materialidad vs. informacionalidad". 
individuos particulares (Manovich 2009). Si la ciudad anterior al siglo XXI fue planeada por una élite y estructurada en base a una taxonomía jerárquica que padece síntomas de agotamiento ${ }^{14}$, la ciudad del entorno aumentado la construyen los propios usuarios.

El declive de la ciudad segregada propuesta por el Movimiento Moderno y defendida en los sucesivos CIAM ha supuesto la revisión de un modelo urbano que debe asumir un tejido social sostenible, también en términos de información. La tecnología dinamita las barreras de contención de usos propios de la ortodoxia conductista y de imposturas positivistas. El tiempo de trabajo se mezcla con el de ocio, el horario laboral ya no se ajusta al reloj solar sino que se diluye en la condición líquida de las estructuras en red. La responsabilidad técnica de lograr un planeamiento urbano de éxito no recaerá exclusivamente en el planeamiento urbanístico de la ciudad masiva del siglo $X X$.

La dimensión digital de la ciudad dinamiza y gestiona las demandas diarias de los ciudadanos. Su versión masiva no basta como soporte social solvente del urbanita contemporáneo. La organización de la información en la ciudad tradicional dispuesta en base al acopio de sustratos independientes ya no resulta operativa en las estructuras urbanas del entorno aumentado, cuya naturaleza simbiótica ha propiciado organizaciones en red que se ajustan al orden rizomático del hipertexto. La polis del entorno aumentado dibuja una red de interacciones con tramos de información cristalina que no lleva a más interpretaciones que la meramente literal, pero a la vez, habilita el enlace con otros flujos de información. Cada ciudadano accede a diferentes nodos de información según sus propios intereses vitales: profesionales, sociales, afectivos, de ocio, etc. Cada uno tiene una experiencia informacional individual que construye un perfil fenomenológico de la ciudad propio e intransferible. Tantas realidades como puntos de vista podamos tener de ella: el ciego, el millonario, el niño, el funcionario, etc.; pero también, tantas como cuantas conexiones podamos establecer con la capa digital de la ciudad. El ciudadano de la Ciudad Aumentada está en manos del dictado de las grandes multinacionales de las telecomunicaciones. La mediación o el acceso a esa extensión urbana tiene un precio: hay muchas Venecias, pero muchas más aumentadas.

\footnotetext{
${ }^{14}$ Por ilustrarlo con algún ejemplo, recuérdense los sucesos desencadenados como protesta social a la implantación de sendos proyectos urbanísticos en el bulevar de Gamonal en Burgos o en la Plaza Taksim en Estambul. "En general nos preocupamos del paisaje urbano, no advirtiendo las amenazas sobre el paisaje humano" (Amann 2011: 43).
} 


\section{Sintonización del paisaje urbano. La ciudad dial y el umbral aumentado}

La percepción depende tanto de las características del medio interpuesto entre el objeto y el sujeto como del órgano sensorial perceptor. La percepción electrónica se supedita, entre otros factores, a la distancia perspectiva y al alcance estimulante de la señal emisora. La percepción del paisaje aumentado no está sujeta a las leyes biológicas de la óptica o de la acústica, pero tampoco ha de someterse a las propias del ciberespacio ${ }^{15}$, donde las acciones de copiar, pegar, subir, bajar, cortar, etc., construyen figuraciones digitales cuya arquitectónica replica parcialmente la del mundo biológico. Es precisamente su condición híbrida -o mejor dicho simbiótica, pues en ningún momento los entornos que lo componen pierden su identidad- la que invita a una reflexión en profundidad acerca de las cualidades arquitectónicas del lugar aumentado y del modo en que éste se habita. Su fundamento tecnológico habilita la presencia de un dato digital en un lugar concreto cuya naturaleza resultará "aumentada", al solaparse en él la capa digital mediante técnicas de geolocalización. Dado el caso, mediante el sistema de referencia global GPS ${ }^{16}$ o similar (figura 2). La percepción de este lugar dual estará sujeta y programada según instrucciones registradas en dicha capa digital, cuyo operativo se activará desde el dispositivo electrónico que el usuario emplea para conectarse al paisaje aumentado. Esta facultad para la acción perceptiva digital se encuentra en la base del concepto de "umbral aumentado"17 o ámbito perceptivo vinculado a un campo de información digital. Cabe aclarar que el umbral aumentado donde se manifiesta la información no necesariamente coincide con la demarcación geográfica donde se produce su georreferencia -donde se "ancla".

Dicho esto, se puede concluir que la disposición de la información en el entorno aumentado estará programada para que se haga perceptible en un tiempo específico, por un agente concreto y desde un dispositivo electrónico particular. Así, la envolvente digital de una arquitectura aumentada puede empezar a ser percibida por un peatón -vista, oída, olida, etc.- a través de su dispositivo electrónico, desde la acera de enfrente del edificio en cuestión o a una distancia mucho mayor si cabe, desde la cual ni siquiera se vea la construcción. Tampoco el tamaño de la información digital tiene que

${ }^{15}$ La tesis Habitar lo irreal. Aproximaciones a una arquitectónica de los mundos virtuales explora este tema con precisión y profundidad (López-Galiacho 2014).

${ }^{16}$ Nótese la variedad de tolerancias en la precisión del posicionamiento de objetos que habilitan los sistemas de posicionamiento global en sus diferentes versiones: civil, comercial o de uso militar.

17 A propósito de la dimensión del umbral aumentado, considérense los conceptos de "proximalidad" y "distalidad" acuñados por Echeverría (1999: 58). 
acomodarse a la escala de la ciudad física ni tiene por qué ser estático, pues su naturaleza transfigurable permite dinamizar la visualización, audición, etc., del contenido digital.

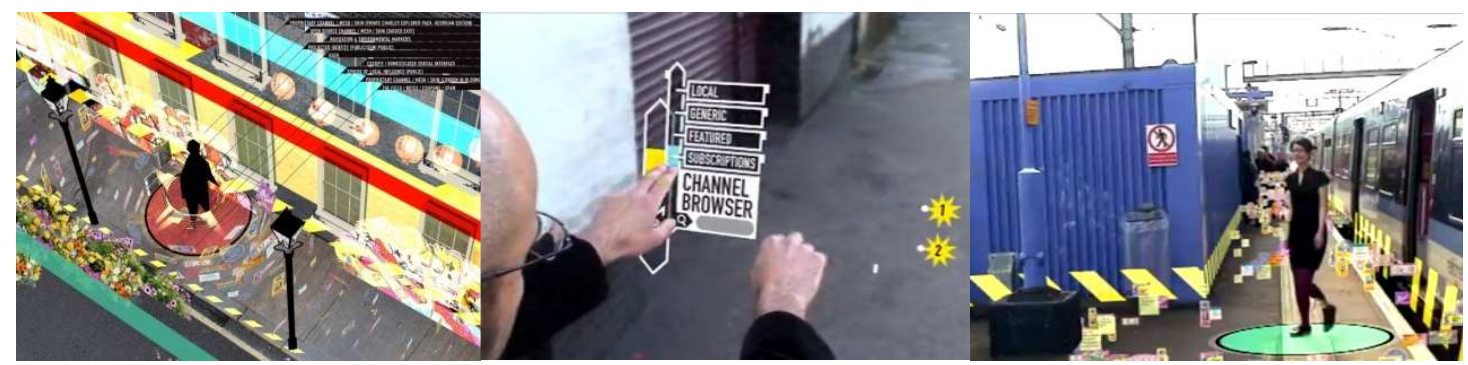

Figura 2. Keiichi Matsuda, Augmented City, 2010.

La lectura de la capa digital se activará en el dispositivo electrónico del individuo; un teléfono, su wearable computing, o un injerto implantando mediante neurocirugía sintonizará la 'ciudad' que prefiera y le sumergirá en la liquidez de la Ciudad Aumentada. Tan sólo debe señalar el punto deseado del dial ${ }^{18}$ para comenzar a vivir en la realidad alternativa. Igual que sucede con otras tecnologías, el número de puntos disponibles del dial aumentado estará supeditado al mercado: si pagas más tienes mayor accesibilidad; si pagas mucho más, dispones del dial completo.

No podemos olvidar que la información que provee el entorno aumentado se integra fácilmente en Internet. Será por tanto un internauta el que elija su experiencia aumentada, aunque como se ha apuntado, esta sea una elección ciertamente condicionada. La carrera por el control de la interacción ya ha comenzado. Las grandes compañías de comunicación se posicionan para resolver la preeminencia de su hegemonía en la Ciudad Aumentada.

La geolocalización digital de la imagen o del sonido o de un olor en un punto concreto de la ciudad dial puede categorizarse según diferentes parámetros. Uno de ellos hace referencia a la naturaleza y a la intensidad de la simbiosis, pues regula el solapamiento de los dos entornos en relación con la percepción del usuario. El grado de convergencia o fusión de los dos entornos determinará así el tipo de entorno aumentado. A continuación se propone la siguiente clasificación topológica al respecto:

${ }^{18}$ Blended Reality Digital Story: The World as I Choose to See It es un proyecto audiovisual del Institute for the Future. El vídeo, cuya dirección se adjunta, muestra la simulación de un paseante que lleva puestas unas gafas aumentadas. Disponible en: http://www.iftf.org/our-work/people-technology/technology-horizons/blendedreality/ [Consulta: 10/09/2013]. 
1) Paisaje aumentado supradigital, o de percepción digital exclusiva. Supone una percepción opaca de la capa digital, tal que se inhabilita la percepción del paisaje físico tradicional, como sucede en el caso de una valla publicitaria, de una pantalla o de unos auriculares. Tal es el modelo de la realidad alternativa que propone Matrix.

2) Paisaje aumentado transdigital, fundamentado en una percepción mixta. La información digital se muestra esta vez total o parcialmente permeable a la percepción del plano que constituye la realidad física. El gradiente de permeabilidad de la capa digital define así mismo la porosidad del entorno aumentado, algo así como su grado de penetración en la cotidianeidad. La porosidad y la compacidad son atributos propios del espacio topológico ${ }^{19}$ del entorno aumentado.

3) Paisaje aumentado ubicuo. Es el característico de una situación donde únicamente se perciba la realidad física, a pesar de que la capa digital esté asociada a ella. La información digital permanece oculta tras el paisaje masivo. Sirva de ejemplo un espacio monitorizado que toma información de un lugar mediante un campo de sensores.

William J. Mitchell se refirió al paisaje supradigital como a un "tipo de solipsismo alimentado electrónicamente [...] extraordinariamente inútil" (Mitchell 1999: 40). Mitchell optó por una nueva arquitectura híbrida que integrara la percepción digital con la física, como en el caso del paisaje aumentado transdigital. Una realidad física capaz de asumir sinergias digitales y viceversa, donde la extensión electrónica acompañara y potenciara ecosistemas más eficientes y flexibles para el usuario:

Es posible por ejemplo, incorporar prismas en las piezas oculares de las gafas de realidad virtual, lo que sobre-impresiona gráficos de ordenador en la escena circundante, de manera que da la impresión de que los objetos virtuales en tres dimensiones se mezclan con los físicos.

Estos escenarios urbanos que hoy comienzan a ser experimentados en casos reales han sido teorizados mucho antes. En el prólogo de e-topía, de nuevo Mitchell resumía la historia de la ciudad en base a los adelantos tecnológicos, reflexionando sobre su modelo de consumo. Finalmente concluía de este modo:

${ }^{19}$ Tómese como ejemplo de información topológica el mapa del metro de Madrid. 
La historia se repite, esta vez porque el sistema de suministro de información ha cambiado. Antes, teníamos que ir a lugares para cosas; íbamos a trabajar, a casa, al teatro, a conferencias, al bar de la esquina o salíamos por ahí, sin más. Ahora tenemos tuberías para bits, redes digitales de gran capacidad para transportar información cuando y donde queramos. Esto nos permite hacer muchas cosas sin tener que ir a ninguna parte; por el contrario, los antiguos lugares de reunión ya no nos atraen; las organizaciones se fragmentan y dispersan; los centros urbanos no se mantienen; da la impresión de que la vida pública va desapareciendo. (Mitchell 1999: 3)

Como otros muchos analistas, Mitchell atisbó una involución de la vida social y por tanto de la ciudad entendida como foro social: ¿para qué vamos a salir de casa si lo podemos hacer desde casa con internet?

La respuesta será muy distinta dependiendo de si contesta un usuario desde su inmersión ciberespacial en internet o si lo hace un individuo conectado a la red que habita la Ciudad Aumentada. El solapamiento de la dimensión digital en el tejido urbano viene a inaugurar una reflexión sobre la inmediatez de una propiedad específica de los archivos digitales: la capacidad transfigurable de la información en soporte bit -multiplicada en la computación cuánticay sus efectos en el proceso de fusión con la ciudad tradicional, estática, permanente. La adaptabilidad del paisaje aumentado, entendida en parámetros formales que se refieran a su envolvente digital, es una cualidad que sin duda facilitará la gestión del cambio. La regeneración de muchos lugares vacíos de programa, porque su condición arquitectónica no permita por ejemplo albergar una actividad demandada, puede resultar mucho más económica y sostenible en el formato aumentado de la ciudad. Dicha adaptabilidad puede derivar en perfectibilidad, o capacidad perfectible de la ciudad por sus ciudadanos. Los usuarios de la Ciudad Aumentada podrán personalizar la capa líquida del paisaje aumentado de modo que se ajuste mejor a su necesidad, circunstancia que ya tienen en cuenta todas las compañías del mercado de las TIC.

Asimismo, el fundamento simbiótico de la Ciudad Aumentada puede alentar una revisión de la especialización de las técnicas constructivas, superada la estética estática de los lugares off line predigitales. Esta impostura bien pudiera ir en beneficio de otros aspectos ecológicos y energéticos que mejorasen la sostenibilidad de las ciudades. La multiplicación dimensional espacio-temporal es una competencia de la Ciudad Aumentada capaz de fragmentar el orden escalar de su naturaleza para adaptarlo al servicio de sus habitantes, de sus visitantes, o de las administraciones que la gestionen. La Ciudad Aumentada no sólo ofrece al ciudadano una propuesta renovadora sino una acción "customizante". Frente al solipsismo del 
ciberespacio, la Ciudad Aumentada multiplica la capacidad de comunicación de la ciudad histórica y actualiza el marco relacional urbano del individuo del siglo XXI.

El abandono de la virtualidad ciberespacial de las pantallas se corresponde con la aparición de los entornos aumentados a finales del siglo $\mathrm{XX}$, una vía tecnológica alternativa donde la computación evoluciona al son de las sinergias entre bits y átomos, concurrencia que puede denominarse como "deriva tecnológica convergente" (Roig Segovia 2014: 235). Fruto de ella han germinado recientemente las tecnologías de impresión 3D o el Internet de las cosas, cuyo desarrollo afecta de manera global a la economía, la salud, la comunicación o la industria, y anticipa cambios en el proceso de construcción de la Ciudad Aumentada.

\section{Bibliografía}

AMANN, Atxu (2011): El espacio doméstico: la mujer y la casa. Buenos Aires: Nobuko.

AUGÉ, Marc (1995): Non-places: Introduction to an Anthropology of Supermodernity. London / New York: Verso.

BAUMAN, Zygmunt (2001): La sociedad individualizada. Madrid: Cátedra.

BODDY, Trevor (2004): "New Urbanism: 'The Vancouver Model' (Speaking of Places)" [en línea]. Places, vol. 16, núm. 2, p. 14. En: http://escholarship.org/uc/item/0ns9f0b0 [Consulta: 20/07/2014].

BORJA, Jordi; y MUXI, Zaida (2001): El espacio público: ciudad y ciudadanía. Barcelona: Diputación de Barcelona.

COLOMINA, Beatriz (2006): La domesticidad en Guerra. Barcelona: Actar.

ECHEVERRÍA, Javier (1999): Los Señores del aire: Telépolis y El Tercer Entorno. Madrid: Destino.

INTERNATIONAL DATA CORPORATION (2008): The Diverse and Exploding Information Universe, 2008 [en línea]. En: http://www.emc.com/leadership/programs/digital-universe.htm [Consulta: 28/11/2014].

LÓPEZ-GALIACHO, Emilio (2014): Habitar lo irreal. Aproximaciones a una arquitectónica de los mundos virtuales. Tesis doctoral no publicada. Universidad Politécnica de Madrid, Escuela Superior de Arquitectura, Dpto. de Ideación Gráfica, Madrid.

MANOVICH, Lev (2009): "Cultural Analytics: Visualizing Cultural Patterns in the Era of 'More Media'". Domus.

MATSUDA, Keiichi (2010): DOMESTI/CITY: The Dislocated Home in Augmented Space [en línea]. En: http://www.keiichimatsuda.com/kmatsuda_domesti-city.pdf [Consulta: 04/08/2012].

McLUHAN, Marshall (1967): Understanding Media: The Extensions of Man. Cambridge, Massachusetts: The MIT Press.

MITCHELL, William J. (1999): e-topía. Cambridge, Massachusetts: The MIT Press.

MITCHELL, William J. (2004): Me++; The Cyborg Self and the Networked City. Cambridge, Massachusetts: The MIT Press. 
MVRDV-UWM (2007): Skycar City. Barcelona: Actar.

NEGROPONTE, Nicholas (1998): "Beyond Digital" [en línea]. Wired, vol. 6, núm. 12. En: http://web.media.mit.edu/ nicholas/Wired/WIRED612.html [Consulta: 20/06/2011].

ROIG SEGOVIA, Eduardo (2014): El Entorno Aumentado. Imperativo informacional para una ecología digital de lo arquitectónico. Tesis doctoral no publicada. Universidad Politécnica de Madrid, Escuela Superior de Arquitectura, Dpto. de Ideación Gráfica, Madrid.

RUEDA, Salvador (2012): "El urbanismo ecológico: un nuevo urbanismo para abordar los retos de la sociedad actual" [en línea], en Cátedra Municipios Sostenibles, Universitat Politècnica de València. En: http://www.upv.es/contenidos/CAMUNISO/info/UrbanismoEcologicoS Rueda.pdf [Consulta: 20/10/2014].

SENNETT, Richard (1970): The uses of disorder: Personal Identity and City Life. New York: Norton.

SENNETT, Richard (2008): El artesano. Barcelona: Anagrama.

VIDAL, Tomeu; y POL, Enriq (2005): "La apropiación del espacio: una propuesta teórica para comprender la vinculación entre las personas y los lugares". Anuario de Psicología, vol. 36, núm. 3, pp. 281-297. 